\title{
Effets combinés de l'aluminium, du fer, et du phosphore sur l'absorption d'ions et le rendement du riz (Oryza sativa $L$ ) en solution nutritive
}

\author{
Vo Dinh Quang 1, Tang Van Hai 2, E Tombo Kanyama 2, JE Dufey 2* \\ 1 Institute of soils and fertilizers, 37 Ben Chuong Duong, Q1, Ho Chi Minh Ville, Viêt-nam \\ 2 UCL-sciences du sol, place Croix du Sud 2/10, B-1348 Louvain-la-Neuve, Belgique
}

(Reçu le 12 septembre 1995; accepté le 22 février 1996)

\begin{abstract}
Résumé - Des cultures de riz (var IR60A) ont été menées en phytotron dans des solutions nutritives contenant différentes concentrations en aluminium (Al) $(74,185,370 \mu \mathrm{M})$, Fer $(\mathrm{Fe})(18,125,357 \mu \mathrm{M})$, et phosphore $(\mathrm{P})(28,70$, $140 \mu \mathrm{M}$ ). Les 27 combinaisons de concentrations ont été expérimentées en double. Les vitesses d'absorption d'ammonium et de $\mathrm{P}$ ont été mesurées en cours de culture et notamment au $45^{\mathrm{e}}$ jour (tallage) et au $80^{\mathrm{e}}$ jour (floraison). La production de biomasse aérienne et racinaire et le rendement en grains ont été déterminés à maturité. L'addition de $P$ ne modifie pas la biomasse végétative, mais permet d'augmenter les rendements en grain, ou, en d'autres termes, de diminuer les chutes de rendement dues à $\mathrm{Al}$ et à $\mathrm{Fe}$. Des régressions multiples pas-à-pas montrent que le rendement en grain est relié de façon très hautement significative $\left(r^{2}=0,86\right)$ aux concentrations en $\mathrm{Al}^{3+}$ et $\mathrm{Fe}^{2+}$ calculées par un programme de spéciation chimique des solutions ; l'addition de $\mathrm{P}$ entraîne la formation de complexes ioniques avec $\mathrm{Al}$, ce qui réduit la concentration en $\mathrm{Al}^{3+}$. Le rendement à maturité (108 jour) est également étroitement corrélé $\left(r^{2}=0,75\right)$ avec la vitesse d'absorption d'ammonium au tallage (45e jour), tous traitements Al-Fe-P confondus.
\end{abstract}

Oryza sativa $\mathrm{L}$ = riz / phosphore / aluminium / fer / culture hydroponique / absorption d'ions

Summary - Combined effects of aluminium, iron and phosphorus on ion uptake and yield of rice plants (Oryza sativa $\mathrm{L}$ ) in nutrient solution. Rice (var IR60A) was grown in nutrient solutions at different concentrations of aluminium $(74,185,370 \mu \mathrm{M})$, iron $(18,125,357 \mu \mathrm{M})$, and phosphorus $(28,70,140 \mu \mathrm{M})$. The 27 combinations of concentrations were tested in duplicate. The uptake rates of ammonium and $P$ were measured at different growth stages, namely after 45 days (tillering) and 80 days (flowering). Shoot and root biomass and grain yield were measured at maturity (108 days). Phosphate uptake rate did not affect plant biomass, but increased grain yield, ie, it alleviated the yield losses due to increased $\mathrm{Al}$ and $\mathrm{Fe}$ concentrations. Stepwise multiple regression showed that the grain yield was strongly correlated $\left(\mathrm{r}^{2}=0.86\right)$ to the $\mathrm{Al}^{3+}$ and $\mathrm{Fe}^{2+}$ concentrations as calculated by a speciation program; increased $P$ uptake rates result in the formation of Al-P complexes that reduce the $A^{\beta+}$ concentration. The grain yield at maturity was also closely related $\left(r^{2}=0.75\right)$ to the uptake rate of ammonium at tillering: a single relationship was observed for all Al-Fe-P treatments.

Oryza sativa $L$ = rice / phosphorus / aluminium / iron / solution culture / ion uptake

* Correspondance et tirés à part. 


\section{INTRODUCTION}

La toxicité aluminique est un facteur souvent invoqué pour expliquer les chutes de rendement du riz en sols acides (Fageria et al, 1988 ; Tang Van Hai et al, 1989, 1993 ; Singh et al, 1991 ; Tran, 1991). L'augmentation du $\mathrm{pH}$ due aux réactions de réduction lors de la submersion des rizières peut diminuer la solubilité de l'aluminium, mais cette opération induit alors une mise en solution d'ions ferreux dont la toxicité au-delà de certaines concentrations est également bien connue (Ponnamperuma, 1972 ; Ottow et al, 1993 ; Vo Dinh Quang et Dufey, 1995 ; Vo Dinh Quang et al, 1995). En pratique, le potentiel redox des rizières submergées varie très largement dans le temps selon la conduite des irrigations et les périodes où les parcelles sont exondées ; il varie également à l'échelle du microsite puisque le riz entretient dans sa rhizosphère des conditions d'oxydation différentes de celles qui règnent dans la masse du sol. En conséquence, comme Moore et al (1990), Moore et Patrick (1993), Genon et al (1994) I'ont identifié, des activités élevées en $\mathrm{Al}$ et/ou en $\mathrm{Fe}$ comptent parmi les facteurs majeurs limitant les rendements du riz sur sols acides.

Des études ciblées de toxicité aluminique et de toxicité ferreuse en conditions de plein champ, et même en pots, conduisent souvent à des résultats ambigus, puisqu'il n'est guère possible d'imposer au sol des traitements menant à des concentrations en $\mathrm{Al}$ et $\mathrm{Fe}$ solubles préalablement décidées. II est, de plus, impossible de faire varier les concentrations de $\mathrm{Fe}$ et d'Al dans la solution du sol indépendamment l'une de l'autre, la raison principale étant que $\mathrm{pH}$ et potentiel redox ne fluctuent pas indépendamment l'un de l'autre en sols inondés. Cette contrainte inhérente au comportement physicochimique particulièrement complexe de l'Al et du Fe dans les sols renforce l'intérêt d'études en solutions nutritives pour cerner avec plus de précision les conséquences de l'excès d'Al et/ou de Fe sur la croissance du riz. C'est cette démarche qui a été adoptée dans l'étude que nous rapportons ici.

Les effets dommageables de l'excès de $\mathrm{Fe}$ et/ou d'Al soluble ont très souvent été associés à une perturbation de la nutrition phosphatée, de sorte que des apports de phosphore constituent l'une des mesures fréquemment préconisées pour mitiger la toxicité aluminique et/ou ferreuse (van Breemen et Moormann, 1978 ; Alva et al, 1986 ; Tang Van Hai et al, 1989 ; Tan et Keltjens, 1990 ; Ottow et al, 1993). En conséquence, il a paru opportun d'inclure l'effet du phosphore dans cette étude sur l'Al et le $\mathrm{Fe}$, d'autant plus que la littérature contient très peu d'information sur l'interaction $\mathrm{Al}-\mathrm{Fe}-\mathrm{P}$ chez le riz en conditions bien contrôlées.

\section{MATÉRIEL ET MÉTHODES}

Les cultures se sont déroulées en chambre climatisée à $25^{\circ} \mathrm{C}$ et $90 \%$ d'humidité relative la nuit, et à $30{ }^{\circ} \mathrm{C}$ et $70 \%$ d'humidité relative le jour. La photopériode était de $12 \mathrm{~h} . \mathrm{j}^{-1}$ avec une intensité lumineuse de $250 \mu \mathrm{mol} . \mathrm{m}^{-2} \cdot \mathrm{s}^{-1}$. Après 7 jours de germination sur tamis en nylon dans l'eau déminéralisée aérée, cinq plantules de riz de variété IR60A ont été repiquées et maintenues par du coton dans les orifices de disques en PVC de $7 \mathrm{~cm}$ de diamètre. Les plantules ont ensuite été placées, par groupes de 12 disques, sur des bacs de $20 \mathrm{~L}$ contenant une solution nutritive dont la composition était la suivante $(\mu \mathrm{M}): \mathrm{NH}_{4} \mathrm{NO}_{3} 1785, \mathrm{KCl}$ $609, \mathrm{KH}_{2} \mathrm{PO}_{4} 32, \mathrm{CaCl}_{2} 250, \mathrm{MgSO}_{4} 206$, FeEDTA 18 + oligoéléments $(\mathrm{B}, \mathrm{Mn}, \mathrm{Zn}, \mathrm{Cu}, \mathrm{Mo}$ : solution mère de Hoagland à raison de $\left.1 \mathrm{~mL} . \mathrm{L}^{-1}\right)$. Le $\mathrm{pH}$ de cette solution en l'absence de plantes était de 5,15. Les solutions ont été renouvelées tous les 2 jours. Cette période de préculture destinée à produire de jeunes plantes vigoureuses a duré 1 mois.

Pour l'expérience proprement dite comportant les traitements $\mathrm{Al}-\mathrm{Fe}-\mathrm{P}$, chaque disque de cinq plantes était placé sur des pots de culture de $1,2 \mathrm{~L}$ où la solution, préparée le jour même, était renouvelée quotidiennement. Vingt-sept types de solutions (croisement de trois doses par élément étudié) ont été utilisées. Les concentrations des éléments différant de celles de la préculture décrite ci-dessus étaient les suivantes:

$-\mathrm{Al}-\mathrm{Al}_{2}\left(\mathrm{SO}_{4}\right)_{3}: 74-185-370 \mu \mathrm{M}$, soit $2-5-10 \mathrm{mg}$ Al. $L^{-1}$

- Fe-FeSO $4: 18-125-357 \mu \mathrm{M}$, soit $1-7-20 \mathrm{mg}$ Fe.L-1;

- $\mathrm{P}-\mathrm{KH}_{2} \mathrm{PO}_{4} ; 28-70-140 \mu \mathrm{M}$, soit $0,87-2,17-$ $4,34 \mathrm{mg}$ P.L.-1.

La gamme de concentrations en Al est celle qui avait été expérimentée dans nos études antérieures (Tang Van Hai et al, 1989, 1993). Le niveau le plus élevé en Al est de l'ordre de grandeur des concentrations que l'on peut trouver dans les solutions de sols acides à un $\mathrm{pH}$ légèrement inférieur à 4 (Ponnamperuma, 1977). Ce même auteur rapporte également des concentrations en $\mathrm{P}$ qui peuvent s'élever à $4 \mathrm{mg} \cdot \mathrm{L}^{-1}(124 \mu \mathrm{M})$ après quelques semaines de submersion. Dans des extraits de pâtes saturées de sols acides, Naidu et al (1991) mesurent des concentrations en $\mathrm{P}$ allant de 0,58 à $10,6 \mathrm{mg} \cdot \mathrm{L}^{-1}(18$ à $330 \mu \mathrm{M})$. Même si des concentrations aussi élevées en $P$ ne sont pas fréquentes, il a paru utile d'étendre notre gamme jusqu'à de telles valeurs pour voir comment, dans l'absolu, ce niveau de $\mathrm{P}$ pouvait pallier les 
effets de l'excès d'Al. Les concentrations en Fe ont été fixées sur la base d'essais préliminaires incluant une collection de variétés mentionnées dans un article précédent (Tang Van Hai et al, 1993) ; les concentrations appliquées ne sont pas particulièrement élevées par rapport à ce que l'on peut trouver dans les solutions de sols submergés ; une discussion quant à la pertinence des concentrations en Fe mesurées in situ est abordée dans la section suivante. Les valeurs du $\mathrm{pH}$ des solutions préparées se trouvaient dans la gamme de 3,1 à 3,8 (tableau 1). Ces faibles valeurs résultent principalement de l'hydrolyse des ions aluminiques; on rencontre fréquemment cette situation dans les sols sulfatés acides (Dent, 1986), contexte dans lequel la présente étude a été entreprise. La concentration en $\mathrm{KCl}$ dans les solutions nutritives a été ajustée en fonction du traitement $P$ pour maintenir une concentration de $K$ constante de $641 \mu \mathrm{M}$; FeEDTA a été supprimé. Pour la simplicité des notations ultérieures, les traitements seront désignés par les symboles Al1, Al2, Al3 pour les concentrations croissantes en Al, par les symboles $\mathrm{Fe} 1, \mathrm{Fe} 2, \mathrm{Fe} 3$ pour les concentrations croissantes en $\mathrm{Fe}$, et par les symboles P1, P2, P3 pour les concentra- tions croissantes en $P$. L'expérience a été réalisée en double.

Les cultures ont été menées jusqu'à maturité, soit au $108^{e}$ jour après la germination. Le rendement en grain a été mesuré, ainsi que la masse de matière sèche des racines et des parties aériennes après passage à l'étuve à $80^{\circ} \mathrm{C}$. Pendant la période de culture, des mesures d'ammonium, de $\mathrm{P}$, et de $\mathrm{Fe}$ dans les solutions après un jour de contact avec les plantes ont été réalisées à divers moments, ces dosages étant également effectués sur des solutions sans plantes afin de détecter d'éventuelles évolutions non contrôlées. Nous ne rapportons ici que les mesures relatives à deux stades critiques de l'élaboration du rendement : le tallage ( $45^{\mathrm{e}}$ jour) et la floraison ( $80^{\mathrm{e}}$ jour). L'ammonium a été dosé par électrode spécifique (modèle Orion 95-12 sur l'ion-analysateur Orion Research 701). Le phosphore a été dosé par la méthode de Watanabe et Olsen (1965). Le fer à l'état ionique réduit $\mathrm{Fe}^{2+}$ dans la solution a été dosé par la méthode à l'orthophénanthroline (Yoshida et al, 1976). Cette technique de dosage sélectif du fer à l'état réduit a permis de vérifier que l'oxydation du Fe-II en Fe-III

Tableau I. Concentrations totales de Fe, Al et $\mathrm{P}$ dans les solutions nutritives et formes principales sous lesquelles se présentent ces éléments, calculées par un programme de spéciation chimique.

Trait a Concentration totale $(\mu \mathrm{M}) \quad \mathrm{pH}$

Al $\mathrm{Fe} \quad P$

111

112

113

121

122

123

131

132

133

211

212

213

221

222

223

231

232

233

311

312

313

321

322

323

331

332

333

\section{P}

$\begin{array}{rrr}74 & 18 & 28 \\ 74 & 18 & 70 \\ 74 & 18 & 140 \\ 74 & 125 & 28 \\ 74 & 125 & 70 \\ 74 & 125 & 140 \\ 74 & 357 & 28 \\ 74 & 357 & 70 \\ 74 & 357 & 140 \\ 185 & 18 & 28 \\ 185 & 18 & 70 \\ 185 & 18 & 140 \\ 185 & 125 & 28 \\ 185 & 125 & 70 \\ 185 & 125 & 140 \\ 185 & 357 & 28 \\ 185 & 357 & 70 \\ 185 & 357 & 140 \\ 370 & 18 & 28 \\ 370 & 18 & 70 \\ 370 & 18 & 140 \\ 370 & 125 & 28 \\ 370 & 125 & 70 \\ 370 & 125 & 140 \\ 370 & 357 & 28 \\ 370 & 357 & 70 \\ 370 & 357 & 140\end{array}$

Répartition des espèces $(\mu M)$

$\mathrm{Al}+\mathrm{AlSO}_{4}+\mathrm{AlHPO}_{4}+\mathrm{Fe}^{2}+\mathrm{FeSO}_{4}+\mathrm{Al}$-autres Fe-autres P-autres

3,83
3,70
3,63
3,77
3,65
3,60
3,76
3,65
3,58
3,63
3,65
3,40
3,61
3,48
3,37
3,59
3,50
3,65
3,63
3,65
3,40
3,58
3,21
3,16
3,45
3,32
3,09

$\begin{array}{rrrr}37 & 7 & 27 & 17 \\ 5 & 2 & 66 & 17 \\ 0 & 0 & 73 & 16 \\ 37 & 7 & 27 & 121 \\ 6 & 1 & 66 & 121 \\ 0 & 0 & 73 & 118 \\ 38 & 6 & 27 & 348 \\ 6 & 1 & 66 & 347 \\ 0 & 0 & 73 & 340 \\ 120 & 32 & 27 & 17 \\ 87 & 24 & 69 & 17 \\ 36 & 10 & 137 & 17 \\ 121 & 32 & 27 & 120 \\ 89 & 24 & 69 & 120 \\ 36 & 10 & 137 & 120 \\ 123 & 30 & 27 & 344 \\ 90 & 22 & 69 & 344 \\ 36 & 9 & 137 & 343 \\ 243 & 91 & 27 & 17 \\ 212 & 81 & 69 & 16 \\ 163 & 64 & 139 & 16 \\ 246 & 89 & 27 & 118 \\ 214 & 83 & 69 & 118 \\ 163 & 66 & 138 & 118 \\ 251 & 86 & 27 & 340 \\ 220 & 77 & 69 & 339 \\ 166 & 60 & 139 & 339\end{array}$

0
0
0
3
3
3
8
9
8
1
1
1
4
4
5
12
12
12
1
1
1
6
6
6
17
17
17

$\begin{array}{llr}3 & 1 & 1 \\ 1 & 1 & 4 \\ 1 & 2 & 67 \\ 3 & 1 & 1 \\ 1 & 1 & 4 \\ 1 & 4 & 67 \\ 3 & 1 & 1 \\ 1 & 1 & 4 \\ 1 & 9 & 67 \\ 6 & 0 & 1 \\ 5 & 0 & 1 \\ 2 & 0 & 3 \\ 5 & 1 & 1 \\ 3 & 1 & 1 \\ 2 & 0 & 3 \\ 5 & 1 & 1 \\ 4 & 1 & 1 \\ 3 & 2 & 3 \\ 9 & 0 & 1 \\ 8 & 1 & 1 \\ 4 & 1 & 1 \\ 8 & 1 & 1 \\ 4 & 1 & 1 \\ 3 & 1 & 2 \\ 6 & 0 & 1 \\ 4 & 1 & 1 \\ 5 & 1 & 1\end{array}$

a Numéro de traitement : les chiffres représentent successivement les niveaux de traitement $\mathrm{Al}$, Fe et $\mathrm{P}$. 
était négligeable dans les solutions de contrôle sans plantes après une journée. Les prélèvements journaliers d'ammonium, de $P$, et de Fe par le riz ont été calculés par différence entre les concentrations des solutions de départ et les concentrations après un jour de contact, en tenant compte de l'évapotranspiration.

Deux méthodes statistiques ont été utilisées pour le traitement des résultats : d'une part l'analyse de la variance à trois critères croisés (Anova, modèle fixe), et d'autre part la régression linéaire multiple pas-àpas. Les calculs ont été réalisés grâce au logiciel Statview (Feldman et al, 1988).

\section{RÉSULTATS ET DISCUSSION}

\section{Rendement en grain, et production de biomasse à maturité}

Une vue globale de l'influence des traitements est fournie par le tableau II qui présente l'analyse de la variance à trois facteurs pour le rendement en grain et pour la matière sèche de feuilles et de racines à maturité. Le rendement en grain est très fortement affecté $(p<0,001)$ par les trois facteurs étudiés : $\mathrm{Al}, \mathrm{Fe}$ et $\mathrm{P}$. La masse de racines dépend très fortement du niveau d'Al $(p<0,001)$, et dans une moindre mesure des doses de Fe appliquées $(p=0,047)$. La production de feuilles est très fortement liée $(p<0,001)$ aux traitements $\mathrm{Al}$ et $\mathrm{Fe}$. Les traitements $\mathrm{P}$ n'affectent ni les feuilles, ni les racines. On ne note d'interactions entre facteurs que pour le couple $\mathrm{Al}-\mathrm{Fe}$ sur le rendement en grain et sur la production de feuilles, ainsi qu'une faible interaction $\mathrm{Al}-\mathrm{Fe}-\mathrm{P}$ sur le rendement en grain. Afin de facili- ter la discussion, malgré quelques restrictions de signification résultant des interactions relevées ci-dessus, les moyennes observées pour une concentration donnée de chaque élément étudié (moyennes de neuf traitements) sont présentées au tableau III, ainsi que les moyennes par combinaison de deux traitements (moyennes des résultats obtenus aux trois concentrations de l'élément non considéré).

\section{Effet de l'Al}

L'augmentation de la concentration en Al diminue les rendements moyens en grain. Cette tendance est observée quels que soient les traitements $\mathrm{Fe}$ et $P$ (fig 1). L'Al diminue aussi la masse végétati$v e$, mais dans une proportion moindre que ce que l'on observe pour le rendement en grain ; en effet, alors que, par rapport au traitement Al1, le rendement moyen en grain chute de $50 \%$ au traitement $\mathrm{Al} 3$, cette diminution relative n'est que d'une trentaine de pourcent pour les masses de matière sèche aérienne et racinaire. L'inhibition de la croissance des racines est le symptôme de toxicité aluminique le plus fréquemment relevé dans la littérature (Taylor, 1991; Horst, 1995). Les résultats de notre étude en montrent les conséquences sur la biomasse aérienne et sur le rendement, ce dernier paramètre étant très rarement obtenu dans des expériences en solution nutritive, pour des raisons évidentes de lourdeur expérimentale.

\section{Effet du Fe}

L'augmentation de la concentration en $\mathrm{Fe}$ diminue les rendements moyens en grain et les

Tableau II. Résultats de l'analyse de la variance à trois facteurs (traitements Al, Fe et P) pour le rendement en grain et la matière sèche de racines et de feuilles.

\begin{tabular}{|c|c|c|c|c|c|c|c|}
\hline \multirow[t]{2}{*}{ Facteur } & \multirow[t]{2}{*}{$d d l$} & \multicolumn{2}{|c|}{ Grain } & \multicolumn{2}{|c|}{ Racines } & \multicolumn{2}{|c|}{ Feuilles } \\
\hline & & $\Sigma$ carrés & Valeur $p$ & $\sum$ carrés & Valeurs p & $\sum$ carrés & Valeur $\mathrm{p}$ \\
\hline Al & 2 & 50,8 & $<0,001$ & 9,63 & $<0,001$ & 205,2 & $<0,001$ \\
\hline $\mathrm{Fe}$ & 2 & 39,3 & $<0,001$ & 1,37 & 0,047 & 167,7 & $<0,001$ \\
\hline $\mathrm{P}$ & 2 & 11,2 & $<0,001$ & 0,32 & 0,455 & 5,8 & 0,342 \\
\hline $\mathrm{Al} \times \mathrm{Fe}$ & 4 & 5,7 & $<0,001$ & 0,50 & 0,646 & 89,2 & $<0,001$ \\
\hline$A \mid \times P$ & 4 & 0,4 & 0,535 & 0,86 & 0,388 & 18,0 & 0,169 \\
\hline $\mathrm{Fe} \times \mathrm{P}$ & 4 & 0,1 & 0,911 & 0,67 & 0,509 & 1,7 & 0,956 \\
\hline $\mathrm{Al} \times \mathrm{Fe} \times \mathrm{P}$ & 8 & 2,9 & 0,014 & 0,34 & 0,986 & 9,7 & 0,867 \\
\hline \multirow[t]{2}{*}{ Erreur } & 27 & 3,2 & & 5,39 & & 69,7 & \\
\hline & & $\Sigma=113,4$ & & $\Sigma=19,08$ & & $\Sigma=566,9$ & \\
\hline
\end{tabular}


Tableau III. Rendement en grain (G), poids de racines $(R)$ et poids de feuilles (F) en gramme de MS pour cinq plantes : moyennes pour deux facteurs variables ou un seul facteur variable. Les symboles $A 11, A 12, A 13, F e 1, F e 2, F e 3, P 1$, $\mathrm{P} 2, \mathrm{P} 3$ désignent les traitements de concentrations croissantes en $\mathrm{Al}, \mathrm{Fe}$ et $\mathrm{P}$.

Moyennes par combinaisons de deux facteurs

\begin{tabular}{|c|c|c|c|c|c|c|c|c|c|c|c|c|c|c|c|}
\hline Traitements & $G$ & $R$ & $F$ & Traitements & $G$ & $R$ & $F$ & Traitements & $G$ & R & $F$ & Traitements & $G$ & $R$ & $F$ \\
\hline $\mathrm{Al} 1 \mathrm{x} \mathrm{Fe} 1$ & 5,02 & 3,02 & 14,72 & Al1 $\times \mathrm{P} 1$ & 4,14 & 3,08 & 15,81 & Fe1 $x P_{1}$ & 3,75 & 2,62 & 15,04 & Al1 & 4,66 & 3,00 & 15,21 \\
\hline Al1 $x$ Fe2 & 5,23 & 3,08 & 16,25 & $\mathrm{Al} 1 \times \mathrm{P} 2$ & 4,76 & 3,14 & 14,23 & $\mathrm{Fe} 1 \times \mathrm{P} 2$ & 4,26 & 2,81 & 14,75 & A & 3,88 & 2,66 & 14,61 \\
\hline $\mathrm{Al} 1 \times \mathrm{Fe} 3$ & 3,73 & 2,89 & 14,65 & Al1 $\times$ P3 & 5,06 & 2,77 & 15,58 & $\mathrm{Fe} 1 \times \mathrm{P} 3$ & 4,73 & 2,42 & 15,51 & $\mathrm{Al3}$ & 2,32 & 1,98 & 10,81 \\
\hline $\mathrm{Al} 2 \mathrm{x}$ & 4,29 & 2,66 & 16 , & & 3,26 & 2,65 & 13 & $\mathrm{Fe} 2 \mathrm{x}$ & 3,58 & 2,71 & 13 & & 4,25 & 2,61 & 15,10 \\
\hline $\mathrm{Al} 2 \times \mathrm{Fe} 2$ & 4,33 & 2,80 & 15,48 & $\mathrm{~A} \mid 2 \mathrm{x}$ & 3,86 & 2,73 & 14,83 & $\mathrm{Fe} 2 \times$ & 4,19 & 2,77 & 14,58 & $\mathrm{Fe} 2$ & 4,20 & 2,69 & 14,45 \\
\hline $\mathrm{Al} 2 \times \mathrm{Fe} 3$ & 3,01 & 2,51 & 12,05 & $\mathrm{Al} 2 \times \mathrm{P} 3$ & 4,51 & 2,59 & 15,43 & $\mathrm{Fe} 2 \times \mathrm{P} 3$ & 4,82 & 2,60 & 14,79 & $\mathrm{Fe} 3$ & 2,41 & 2,32 & 11,08 \\
\hline $\mathrm{Al} 3 \mathrm{x}$ & 3,43 & 2,17 & 14,29 & & 1,79 & 1,71 & 10,16 & & 1,86 & 2,11 & 10,54 & & 3,06 & 2,48 & 13,18 \\
\hline $\mathrm{Al} 3 \times \mathrm{Fe} 2$ & 3,03 & 2,20 & 11,61 & $\mathrm{Al} 3 \times \mathrm{P} 2$ & 2,22 & 2,09 & 13,35 & $\mathrm{Fe} 3 \times \mathrm{P} 2$ & 2,39 & 2,38 & 11,07 & $\mathrm{P}$ & 3,06 & 2,48 & 13,18 \\
\hline $\mathrm{Al} 3 \times \mathrm{Fe} 3$ & 0,51 & 1,57 & 6,53 & $\mathrm{~A} \mid 3 \times \mathrm{P} 3$ & 2,96 & 2,14 & 10,92 & $\mathrm{Fe} 3 \times \mathrm{P} 3$ & 2,99 & 2,48 & 11,62 & P3 & 4,18 & 2,50 & 13,98 \\
\hline
\end{tabular}

masses de matière sèche lorsque l'on passe du traitement $\mathrm{Fe} 2$ au traitement Fe3. À nouveau, comme pour l'Al, cet effet est plus marqué pour le rendement en grain que pour la production de biomasse, la diminution de biomasse des racines étant particulièrement faible. La dépression des rendements induite par le traitement $\mathrm{Fe} 3$ est vérifiée à tous les niveaux d'Al et de $P$ (fig 2). La combinaison des effets dépressifs de l'Al et du $\mathrm{Fe}$ conduit à une chute relative de rendement en
Fig 1. Effet de la concentration en $\mathrm{Al}$ dans la solution nutritive sur le rendement en grain : a. pour les trois niveaux de $\mathrm{Fe}$ (moyenne des trois traitements $P$ ), et $\mathbf{b}$. pour les trois niveaux de $P$ (moyenne des traitements $\mathrm{Fe}$ ).

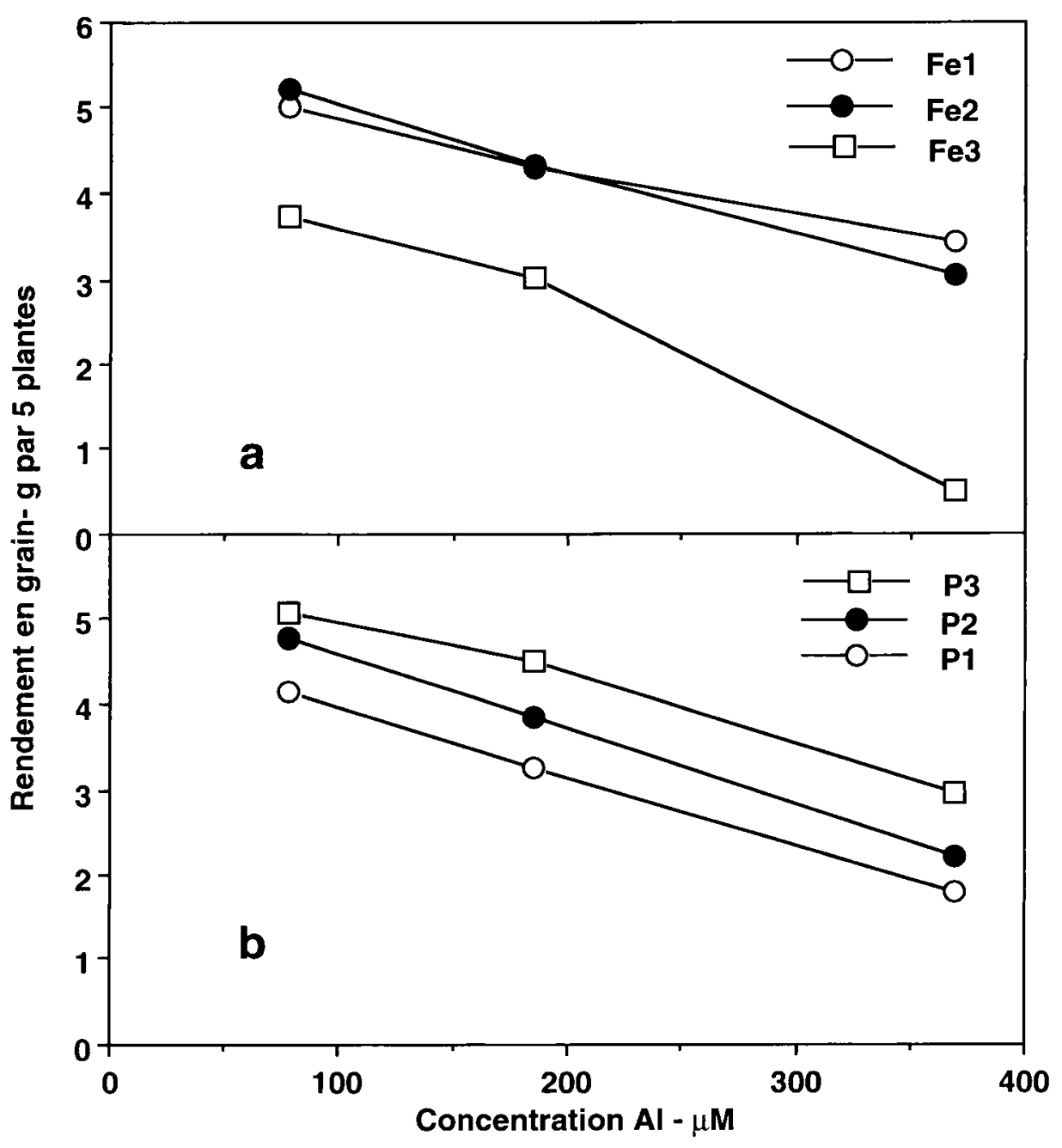




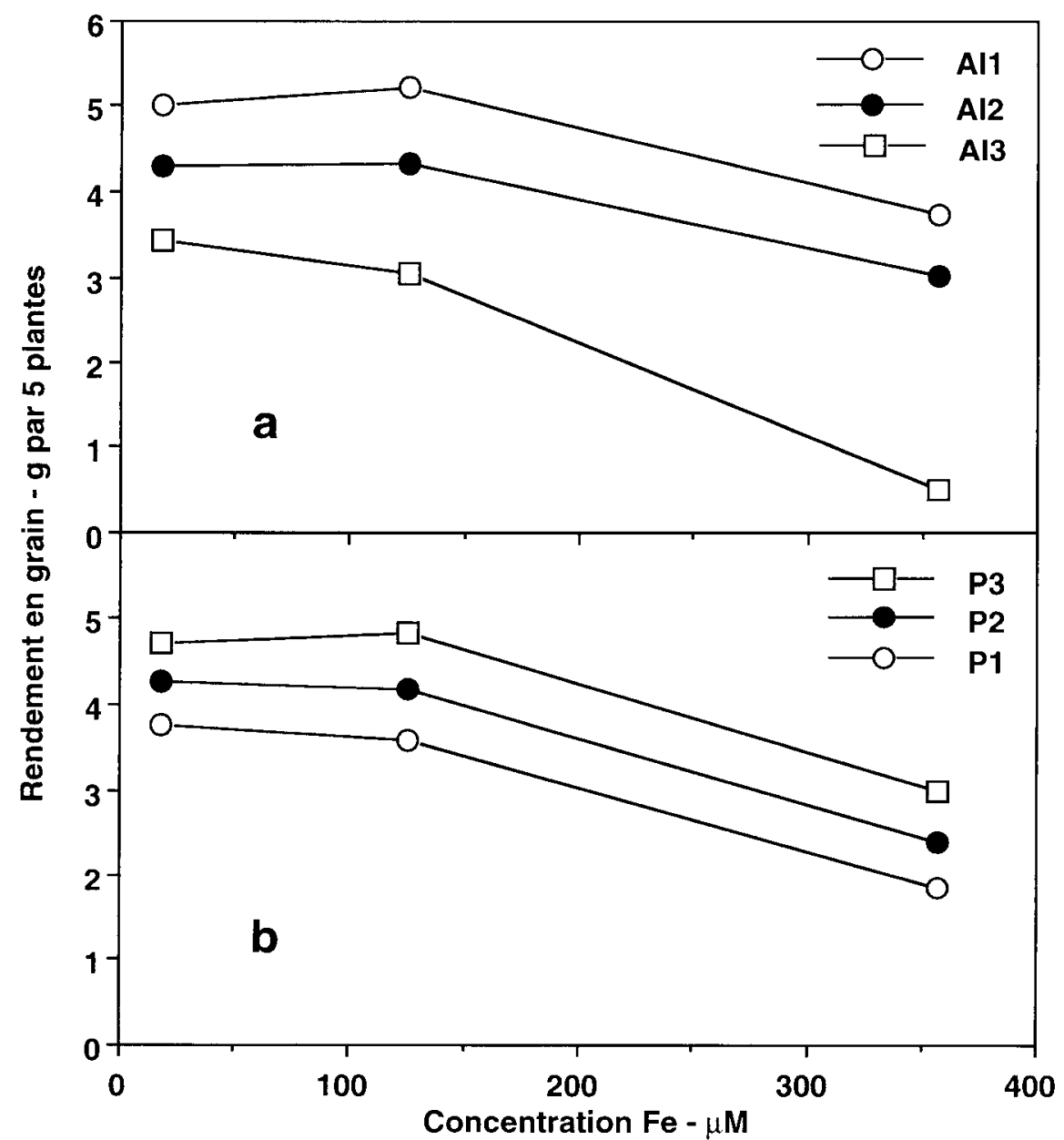

Fig 2. Effet de la concentration en $\mathrm{Fe}$ dans la solution nutritive sur le rendement en grain : a. pour les trois niveaux d'Al (moyenne des trois traitements $P$ ), et $\mathbf{b}$. pour les trois niveaux de $P$ (moyenne des trois traitements $\mathrm{Al}$ ). grain de l'ordre de $90 \%$ par rapport au rendement le plus élevé ; cette réduction est de l'ordre de $60 \%$ pour la biomasse aérienne, et de l'ordre de $50 \%$ pour les racines.

Le seuil de toxicité du $\mathrm{Fe}$ observé dans cette étude (entre les niveaux Fe2, $7 \mathrm{mg} \cdot \mathrm{L}^{-1}$, et $\mathrm{Fe} 3$, $20 \mathrm{mg}^{\left.-\mathrm{L}^{-1}\right)}$ est particulièrement faible par rapport aux observations de terrain de van Breemen et Moorman (1978) et d'Ottow et al (1993) qui fixent ce seuil à des concentrations en Fe dans la solution du sol supérieures à $300 \mathrm{mg} \cdot \mathrm{L}^{-1}$. Nos résultats sont en revanche similaires à ceux qui ont été obtenus par Ishizuka (1961) en solution nutritive. Les causes possibles de désaccord entre résultats obtenus en solution et sur sol sont nombreuses. Par exemple, les concentrations totales en $\mathrm{Fe}$ dans une solution de sol ne peuvent être traduites en concentrations en $\mathrm{Fe}^{2+}$ ionique que moyennant un calcul de spéciation chimique; ce calcul suppose que l'on effectue une analyse détaillée des solutions récoltées, comprenant non seulement toutes les espèces inorganiques, mais également les anions organiques dont le pouvoir complexant est particulièrement élevé. II va de soi que le potentiel redox et le $\mathrm{pH}$ doivent être déterminés dans les conditions prévalant in situ, ce qui est techniquement difficile, et pose de toute façon le problème de la signification physicochimique des mesures potentiométriques effectuées sur des suspensions de sol. Un autre problème est de cerner ce que représentent des mesures sur échantillons moyens par rapport au proche environnement de la racine : des concentrations moyennes élevées en Fe-ll ne signifient pas que cette forme persiste au voisinage immédiat des racines de riz alimentées en oxygène par un aérenchyme. Cette oxydation rhizosphérique qui induit la précipitation du $\mathrm{Fe}$ en oxyhydroxydes de $\mathrm{Fe}$-III est considérée comme un mécanisme d'autoprotection du riz contre la toxicité ferreuse (Ottow et al, 1993).

\section{Effet du P}

Les doses croissantes de phosphore ne modifient pas la production moyenne de biomasse aérienne et racinaire. On note, en revanche, une augmentation très nette du rendement moyen en grain avec la concentration en $\mathrm{P}$ dans les solutions. Comme illustré à la figure 3 , cette observa- 
Fig 3. Effet de la concentration en $P$ dans la solution nutritive sur le rendement en grain : a. pour les trois niveaux d'Al (moyenne des trois traitements $P$ ), et $\mathbf{b}$. pour les trois niveaux de $\mathrm{Fe}$ (moyenne des trois traitements Al).

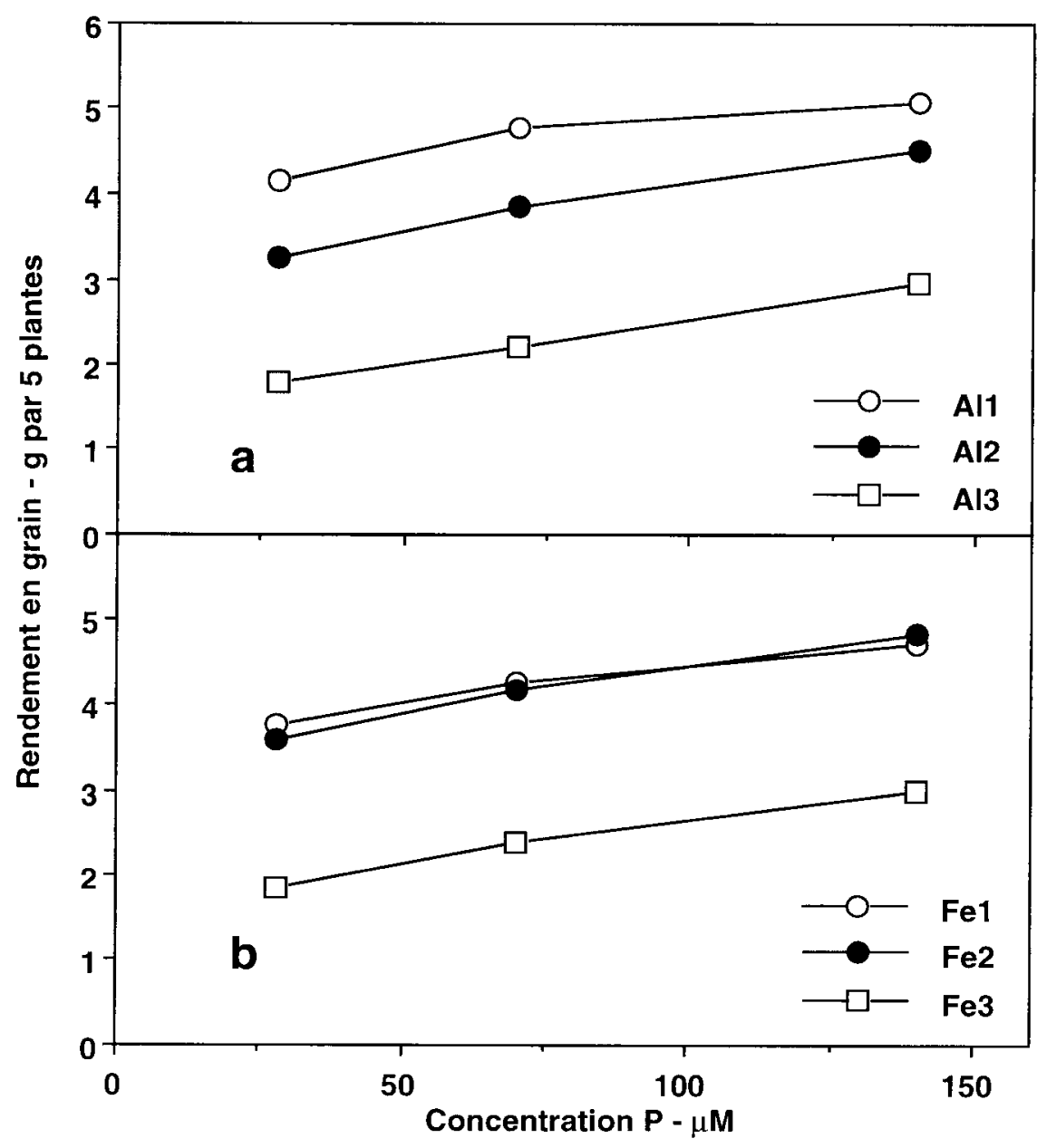

tion est vérifiée à tous les niveaux d'Al et à tous les niveaux de $\mathrm{Fe}$. On note un parallélisme des variations de rendement, traduisant l'absence d'interaction statistique entre les traitements $P$ et les traitements $\mathrm{Al}$ et $\mathrm{Fe}$. L'amélioration des rendements par le $\mathrm{P}$ se superpose simplement aux effets dépressifs de l'Al et du Fe.

L'analyse de la variance (tableau II) montre que les trois facteurs $\mathrm{Al}, \mathrm{Fe}$ et $\mathrm{P}$ interviennent pour une part très importante dans la somme totale des carrés des écarts (101/113), les interactions entre facteurs et l'erreur n'intervenant que pour 12/113. On peut donc tenter d'établir une relation quantitative entre le rendement et les concentrations des trois éléments étudiés. L'équation la plus simple est fournie par une régression linéaire multiple. Une analyse par régression pas-à-pas donne les équations successives suivantes:

$$
\begin{array}{ll}
Y=5,28-0,0079 \mathrm{Al} & r^{2}=0,45 \\
Y=6,24-0,0079 \mathrm{Al}-0,0058 \mathrm{Fe} & r^{2}=0,76 \\
Y=5,47-0,0079 \mathrm{Al}-0,0058 \mathrm{Fe}+0,0098 \mathrm{P} r^{2}=0,86
\end{array}
$$

où $Y$ est le rendement en grain, les concentrations dans la solution nutritive étant exprimées en micromolarité. La valeur du coefficient de variation $r^{2}$ indique qu'une combinaison linéaire des trois facteurs étudiés rend compte de $86 \%$ de la variance du rendement.

\section{Spéciation chimique des solutions et rendements}

Les mécanismes par lesquels le $P$ augmente les rendements, même en présence de $\mathrm{Fe}$ et d'Al, ou, ce qui est une autre façon de voir, les mécanismes par lesquels le $\mathrm{P}$ peut compenser les effets dépressifs du Fe et surtout de l'Al peuvent, bien sûr, être d'ordre physiologique. Néanmoins, l'interprétation du prélèvement d'éléments par une plante suppose la connaissance des formes sous lesquelles se présentent les espèces étudiées dans la solution nutritive et le calcul de leur activité. Le logiciel Species (Barak, 1990) a été utilisé à cet effet. II permet de calculer les concentrations des ions élémentaires et des complexes ioniques à partir des concentrations 
totales des éléments et du $\mathrm{pH}$. Les calculs relatifs aux trois éléments étudiés dans les 27 solutions différentes sont présentés au tableau I. On relève une interaction chimique Al-P particulièrement importante conduisant à la formation du complexe ionique $\mathrm{AlHPO}_{4}{ }^{+}$. Cette espèce se forme systématiquement en une concentration qui est limitée par la plus faible des deux concentrations totales en $\mathrm{Al}$ ou $\mathrm{P}$. En conséquence, d'autres formes d'Al ou de $P$ ne peuvent exister en concentration dépassant la micromolarité que pour le solde de concentration dépassant celle de l'autre espèce. Ainsi, lorsque la concentration totale en Al dépasse celle de $\mathrm{P}$, l'Al non combiné avec $P$ se présente essentiellement sous forme ionique simple $\mathrm{Al}^{3+}$ et sous forme de complexe ionique $\mathrm{AlSO}_{4}{ }^{+}$, dans un rapport molaire de l'ordre de 3 à 4 . Lorsque la concentration totale en $P$ dépasse celle de l'Al (dans trois solutions seulement sur 27), le $P$ non complexé par Al se présente surtout sous forme d'ion orthophosphorique $\mathrm{H}_{2} \mathrm{PO}_{4}^{-}$. Quant au $\mathrm{Fe}$, il est peu impliqué dans des associations ioniques, du moins dans les conditions de nos expériences ; la forme ionique $\mathrm{Fe}^{2+}$ représente toujours plus de $95 \%$ de la concentration totale en Fe.

Ces calculs de spéciation chimique suggèrent que la réduction de la toxicité aluminique par le phosphore puisse être due, entre autres raisons, à la formation des complexes ioniques $\mathrm{AlHPO}_{4}{ }^{+}$, cette association réduisant l'activité $\mathrm{d}^{\prime} \mathrm{Al}^{3+}$ considérée par de nombreux auteurs comme responsable des effets dépressifs (Wagatsuma et Ezoe, 1985 ; Alva et al, 1986 ; Kinraide, 1991). Pour étayer cette hypothèse, on peut procéder à une nouvelle régression multiple pas-à-pas où les paramètres explicatifs du rendement en grain $(Y)$ ne sont plus les concentrations totales en Al, Fe et $P$, mais les concentrations $(\mu M)$ des espèces ioniques établies par le programme species. Les équations successives suivantes sont obtenues :

$$
\begin{array}{ll}
Y=4,90-0,0126 \mathrm{Al}^{3+} & r^{2}=0,56 \\
Y=5,83-0,0125 \mathrm{Al}^{3+}-0,0059 \mathrm{Fe}^{2+} & r^{2}=0,86
\end{array}
$$

L'introduction de la concentration d'une quelconque autre espèce en solution n'améliore plus le coefficient de détermination. On arrive donc à expliquer le même pourcentage de la variance que plus haut en considérant les seules espèces $\mathrm{Al}^{3+}$ et $\mathrm{Fe}^{2+}$, sans plus tenir aucun compte du $\mathrm{P}$. Il est donc tout à fait possible que l'effet bénéfique du $\mathrm{P}$ sur le rendement dans nos solutions soit explicable dans une très large mesure par la complexation Al-P qui diminue l'activité de l'Al ${ }^{3+}$. La complexation Al-sulfate joue aussi un rôle analogue dans la réduction de l'activité $\mathrm{d}^{\prime} \mathrm{Al}^{3+}$ (Tanaka et al, 1987 ; Kinraide et Parker, 1987).

Cette interprétation pose toutefois le problème de la nutrition phosphatée. En effet, comme dans la plupart des solutions, tout le $\mathrm{P}$ est complexé par l'Al, il faut admettre que, tout en détoxifiant I'Al, la forme $\mathrm{AlHPO}_{4}{ }^{+}$est susceptible d'être absorbée par le riz et d'assurer la nutrition phosphatée ; une décomplexation au sein des parois cellulaires, dont l'affinité pour l'Al est bien connue (Taylor, 1991 ; Dufey et al, 1991 ; Horst, 1995), serait une hypothèse à vérifier.

L'effet positif du $P$ sur la réduction de la toxicité ferreuse ne peut pas être lié à un effet strictement physicochimique au sein de la solution nutritive puisque, comme on l'a montré au tableau I, le P s'associe peu au $\mathrm{Fe}$, du moins dans les conditions de nos expériences, où domine la complexation Al-P. Selon certains auteurs (van Breemen et Moorman, 1978 ; Prade et al, 1988 ; Ottow et al, 1993), le $P$ améliorerait le pouvoir oxydant du système racinaire, ce qui contribuerait à bloquer le $\mathrm{Fe}$ à ce niveau et limiterait son transfert vers les parties aériennes. Nos mesures de concentration en $\mathrm{Fe}$ dans les solutions avec ou sans contact avec les plantes montrent que, de fait, l'augmentation des doses de $\mathrm{P}$ induisent une disparition croissante de $\mathrm{Fe}$ de la solution (fig 4), mais il n'est pas possible d'en déduire la part qui est immobilisée au niveau du système racinaire et la part qui est effectivement absorbée et transportée vers les parties aériennes. Cette immobilisation, couplée ou non à la translocation de $\mathrm{Fe}$, est, en revanche, réduite lorsque la concentration en Al augmente. Cela peut résulter tant d'une compétition Al-Fe sur les sites d'échange radiculaires localisés principalement dans les parois, que d'une inhibition de l'activité des transporteurs membranaires par l'Al.

\section{Absorption d'ions}

Le prélèvement journalier de $\mathrm{P}$ et d'ammonium a été suivi à différents stades de la croissance des plantes. Le tableau IV rapporte les résultats de l'analyse de la variance pour ces prélèvements au tallage et à la floraison qui sont deux stades critiques pour l'élaboration du rendement. Le prélèvement de $P$ est toujours le plus influencé par les traitements $P$, aux deux stades étudiés. Les traitements $\mathrm{Al}$ affectent également nettement le prélèvement de $\mathrm{P}$ au tallage et on note des interactions entre tous les facteurs étudiés. Le prélèvement d'ammonium à la floraison n'est lié 
Fig 4. Effet de la concentration en $P$ dans la solution nutritive sur le prélèvement les plantes au tallage pour les trois niveaux de $\mathrm{Fe}$ (moyenne des trois traitements $\mathrm{Al}$ ). journalier de $\mathrm{Fe}$ dans

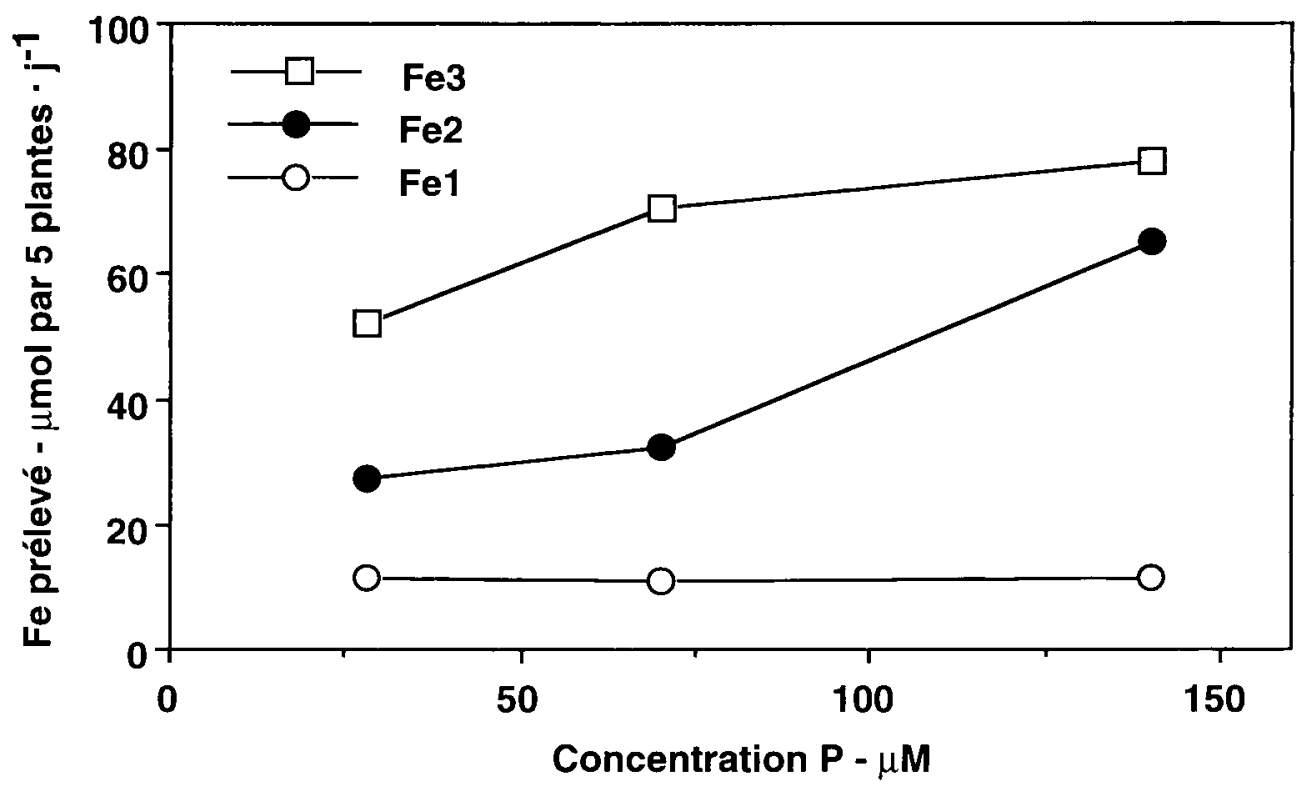

Tableau IV. Résultats de l'analyse de la variance à trois facteurs (traitements Al, Fe et $\mathrm{P}$ ) pour les prélèvements journaliers de $\mathrm{P}$ et d'ammonium au tallage $\left(45^{\mathrm{e}}\right.$ jour) et à la floraison ( $80^{\mathrm{e}}$ jour).

\begin{tabular}{|c|c|c|c|c|c|c|c|c|c|}
\hline \multirow[t]{2}{*}{ Facteur } & \multirow[t]{2}{*}{$d d l$} & \multicolumn{2}{|c|}{$P$ tallage } & \multicolumn{2}{|c|}{$P$ floraison } & \multicolumn{2}{|c|}{$N$ tallage } & \multicolumn{2}{|c|}{$N$ floraison } \\
\hline & & $\sum$ carrés & Valeurp & $\sum$ carrés & Valeur $\mathrm{p}$ & $\sum$ carrés & Valeurp & $\sum$ carrés & Valeurs $\mathrm{p}$ \\
\hline--- & & - & $\ldots$ & 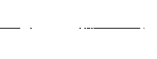 & $\ldots$ & - & -[ & -... & - \\
\hline $\mathrm{Al}$ & 2 & 1,00 & $<0,001$ & 0,02 & 0,698 & 325,8 & $<0,001$ & 17,6 & 0,069 \\
\hline $\mathrm{Fe}$ & 2 & 0,17 & 0,004 & 0,02 & 0,770 & 485,2 & $<0,001$ & 2,0 & 0,719 \\
\hline$P$ & 2 & 3,86 & $<0,001$ & 11,72 & $<0,001$ & 67,9 & $<0,001$ & 32,6 & 0,010 \\
\hline $\mathrm{Al} \times \mathrm{Fe}$ & 4 & 0,42 & $<0,001$ & 1,58 & $<0,001$ & 13,3 & 0,406 & 5,9 & 0,740 \\
\hline$A I \times P$ & 4 & 0,41 & $<0,001$ & 0,07 & 0,660 & 17,6 & 0,270 & 5,1 & 0,787 \\
\hline $\mathrm{Fe} \times \mathrm{P}$ & 4 & 0,18 & 0,014 & 0,16 & 0,280 & 25,2 & 0,129 & 4,3 & 0,835 \\
\hline $\mathrm{Al} \times \mathrm{Fe} \times \mathrm{P}$ & 8 & 0,76 & $<0,001$ & 1,29 & $<0,001$ & 80,0 & 0,013 & 30,3 & 0,297 \\
\hline rreur & 27 & 0,33 & & 0,79 & & 76,5 & & 80,3 & \\
\hline & & $\sum=7,13$ & & $\Sigma=15,65$ & & $\Sigma=1091,5$ & & 178, & \\
\hline
\end{tabular}

qu'aux traitements $P$; en revanche, au tallage, le prélèvement d'ammonium est affecté par les trois facteurs $\mathrm{Al}, \mathrm{Fe}$ et $\mathrm{P}$. À ce stade de végétation, on note que les traitements $\mathrm{Al}$ et $\mathrm{Fe}$ prennent une part très importante dans la somme totale des carrés des écarts, respectivement 325/1101 et $485 / 1101$. L'analyse de la variance des rendements en grain montrait aussi une influence très importante des facteurs $\mathrm{Al}$ et $\mathrm{Fe}$. II est dès lors indiqué d'examiner les relations directes entre rendements et prélèvements d'éléments.

La figure 5 met en évidence une relation intéressante $\left(r^{2}=0,75\right)$ entre le rendement en grain et le prélèvement d'ammonium au $45^{\mathrm{e}}$ jour (tallage), tous traitements confondus. La vitesse d'absorption de $\mathrm{P}$ au $45^{\mathrm{e}}$ jour présente aussi une certaine corrélation avec la production de grain $\left(r^{2}=\right.$ $0,36)$. En revanche les vitesses d'absorption d'ammonium et de $\mathrm{P}$ au $80^{\circ}$ jour (floraison) ne montrent aucune corrélation significative avec le rendement. Les conséquences pratiques de cette observation sont particulièrement intéressantes : les effets dépressifs prévisibles de l'Al et du Fe pourraient être diagnostiqués, avec une fiabilité élevée, à un stade précoce de la culture, en l'occurrence au tallage. Cette suggestion conforte des études antérieures, telles que celles d'Uwasawa et al (1988), Ottow et al (1993) et de Tang Van Hai et al (1993), qui ont mis en évidence le rôle particulièrement critique de la phase de tallage dans l'élaboration du rendement.

\section{CONCLUSIONS}

Cette étude a permis de bien différencier les effets de l'Al, du Fe, et de $\mathrm{P}$ sur la biomasse et le rendement du riz à maturité. De façon générale, 

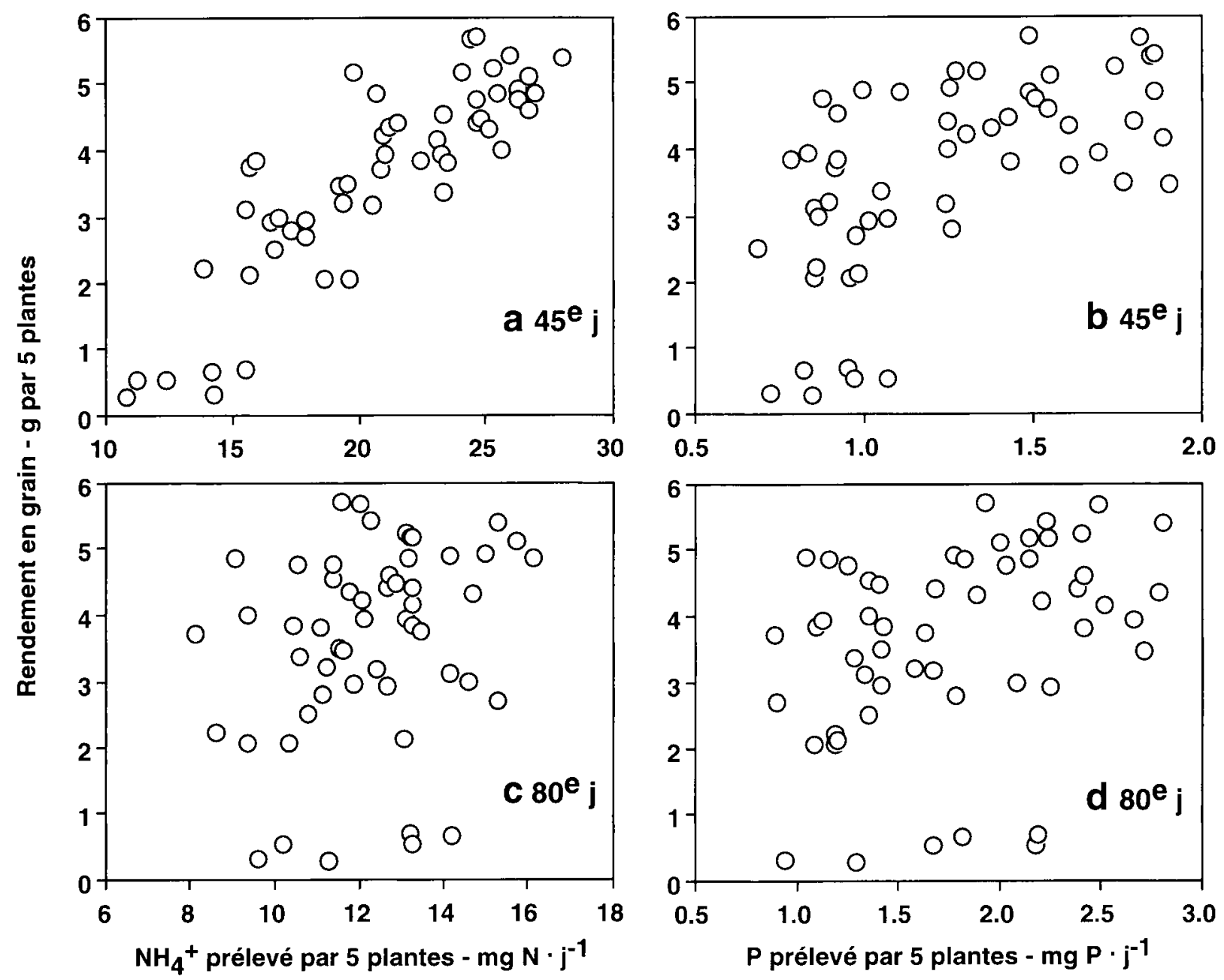

Fig 5. Relation entre le rendement en grain et les prélèvements journaliers d'ammonium et de phosphore au tallage (45e jour, $\mathbf{a}$ et $\mathbf{b})$ et à la floraison $\left(80^{\mathrm{e}}\right.$ jour, $\mathbf{c}$ et d).

les effets sont nettement plus marqués sur le rendement en grain que sur la biomasse : cette observation est particulièrement valable pour le $P$ qui ne provoque pas d'augmentation de biomasse aérienne et racinaire, mais qui diminue efficacement les chutes de rendement dues au $\mathrm{Fe}$ et à I'Al. L'augmentation des concentrations en $\mathrm{Fe}$, dans la gamme étudiée, modifie peu la biomasse racinaire, mais elle diminue la biomasse aérienne et le rendement en grain au-delà d'une concentration en Fe de l'ordre de $10 \mathrm{mg} \cdot \mathrm{L}^{-1}$ en solution. Enfin l'Al diminue les biomasses racinaires et aériennes surtout au-delà d'une concentration de l'ordre de $5 \mathrm{mg} \cdot \mathrm{L}^{-1}$, alors que la chute de rendement est manifeste dans toute la gamme étudiée (2 à $10 \mathrm{mg} \cdot \mathrm{L}^{-1}$ ).

Selon une analyse par régression multiple, les seules concentrations en $\mathrm{Al}^{3+}$ et $\mathrm{Fe}^{2+}$ en solution, calculées par un programme de spéciation chimique, permettent de prédire, avec un très haut niveau de fiabilité, les chutes de rendement en grain observées dans les conditions de nos expériences. Le calcul des concentrations des espèces chimiques en solution permet aussi d'avancer l'hypothèse de l'assimilabilité du $P$ sous forme de complexe avec l'Al, c'est-à-dire sous forme $\mathrm{AlHPO}_{4}{ }^{+}$. La formation de ce complexe ionique contribuerait à expliquer la diminution de la toxicité aluminique par l'addition de $\mathrm{P}$.

Enfin, il est utile de noter que le rendement en grain à maturité est corrélé à l'absorption ionique de $P$ et surtout d'ammonium à un stade végétatif précoce ( $45^{\mathrm{e}}$ jour, c'est-à-dire au tallage). Cette corrélation ne se retrouve plus aux stades ultérieurs, bien que ceux-ci soient plus rapprochés de la maturité.

La transposition de ces données en conditions de champ est, bien entendu, malaisée pour les raisons énoncées dans l'introduction. En sol, la solution peut être réalimentée en $\mathrm{Al}$ et en Fe par les constituants solides, de sorte que l'effet positif des processus de complexation ionique peut 
être mitigé par une dissolution accrue au départ des minéraux. Toutefois, des progrès dans la compréhension des mécanismes de nutrition minérale peuvent manifestement être obtenus par une spéciation chimique fine des solutions. Dès lors, les résultats d'études en solution nutritive trouveront leurs prolongements en sol dans la mesure où des progrès parallèles seront effectués dans la modélisation des processus d'échange et de dissolution/précipitation qui gouvernent la composition de la solution du sol.

\section{REMERCIEMENTS}

Nous remercions le secrétariat à la coopération internationale de l'université catholique de Louvain pour le soutien apporté à notre programme de recherche sur la valorisation des sols acides intertropicaux, notamment par l'octroi d'une bourse de doctorat à Vo Dinh Quang. Nous remercions le professeur G Gérard pour ses conseils en matière d'analyse statistique. Nous avons apprécié les remarques formulées par les lecteurs qui ont revu cet article et qui ont ainsi contribué à en améliorer la qualité scientifique. Cette recherche a été réalisée grâce à un financement de la Commission des Communautés européennes pour la mise en valeur des sols acides du delta du Mékong.

\section{RÉFÉRENCES}

Alva Ak, Edwards DG, Asher CJ, Blamey FP (1986) Effects of phosphorus/aluminium molar ratio and calcium concentration on plant response to aluminium toxicity. Soil Sci Soc Amer J 50, 133-137

Barak P (1990) A spreadsheet program for modeling chemical speciation of soil solution. J Agron Educ 19, $44-46$

Dent D (1986) Acid sulphate soils: a baseline for research and development. International Institute for Land Reclamation and Improvement ILRI, publication 39, Wageningen, the Netherlands

Dufey JE, Drimer D, Lambert I, Dupont P (1991) Composition of root exchange sites in acidic soil solutions. In : Plant Roots and their Environment (BL McMichael, H Persson, eds), Elsevier Science Publishers, Amsterdam, The Netherlands, 31-38

Fageria NK, Baligar VC, Wright RJ (1988) Aluminium toxicity in crop plants. J Plant Nutr 11, 303-319

Feldman DS Jr, Hofmann R, Gagnon J, Simpson J (1988) Statview II. La solution pour l'analyse de données et la représentation graphique. Alpha Système Diffusion, Meylan, France

Genon J, De Hepcée N, Dufey JE, Delvaux B, Hennebert PA (1994) Iron toxicity and other chemical constraints to rice in highland swamps of Burundi. Plant Soil 166, 109-155
Horst WJ (1995) The role of the apoplasm in aluminium toxicity and resistance of higher plants: a review. Z Pflanzenernähr Bodenk 158, 419-428

Ishizuka $Y$ (1961) Inorganic nutrition of rice plant. Part 6. Effect of iron, manganese and copper level in culture solution on yields and chemical composition of the plant. J Soil Sci Manure 32, 97-100

Kinraide TB (1991) Identify of the rhizotoxic aluminium species. In : Plant-Soil Interactions at Low pH (RJ Wright, VC Baligar, RP Murrmann, eds), Kluwer Academic Publishers, Dordrecht, the Netherlands, 717-728

Kinraide TB, Parker DR (1987) Non-phytotoxicity of the aluminium sulfate ion, $\mathrm{AlSO}_{4}{ }^{+}$. Physiologia Plantarum 71, 207-212

Moore PA, Patrick WHJr (1993) Metal availability and uptake by rice in acid sulphate soils. In : Selected Papers of the Ho Chi Minh City Symposium on Acid Sulphate Soils (DL Dent, MEF van Mensvoort, eds), ILRI publication 53, Wageningen, the Netherlands, 205-223

Moore PAJ, Attanandana T, Patrick WH Jr (1990) Factors affecting rice growth on acid sulfate soils. Soil Sci Soc Amer J 54, 1651-1656

Naidu R, Haynes RJ, Gawandar JS, Morrison RJ, Fitzpatrick RW (1991) Chemical and mineralogical properties and soil solution composition of acid soils from the South Pacific Islands. In : Plant-Soil Interactions at Low $p H$ (RJ Wright, VC Baligar, RP Murrmann, eds), Kluver Academic Publishers, Dordrecht, the Netherlands, 43-53

Ottow JCG, Prade K, Bertenbreiter W, Jacq VA (1993) Mécanisme d'intoxication du riz inondé (Oryza sativa L) par les ions ferreux, au Sénégal et en Indonésie. In : Bas-fonds et riziculture (M Raunet, ed), Cirad-Ca, Montpellier, 231-241

Ponnamperuma FN (1976) Physicochemical properties of submerged soils in relation to fertility. The International Rice Research Institute IRRI Research paper series $n^{\circ} 5$, Los Baños, Philippines

Ponnamperuma FN (1972) The chemistry of submerged soils. Advances in Agronomy 24, 26-96

Prade K, Ottow JCG, Jacq V (1988) Excessive iron uptake (iron toxicity) by wetland rice (Oryza sativa L) on an acid sulfate soil in the Casamance/Senegal. In : Selected Papers on the Dakar Symposium on Acid Sulfate Soils, Dakar, Senegal, 1986 (H Dost, ed), ILRI Publication 44, Wageningen, the Netherlands, 150-162

Singh VP, Lantin RS, Neue HU (1991) Management of acid soils for rice production in the Philippines. In: Rice Production on Acid Soils of the Tropics ( $P$ Deturk, FN Ponnamperuma, eds), Institute of Fundamental Studies, Kandy, Sri Lanka, 123-129

Tan K, Keltjens WG (1990) Interaction between aluminium and phosphorus in sorghum plants. I. Studies with the aluminium sensitive sorghum genotype TAM428. Plant Soil 124, 15-23

Tanaka A, Tadano T, Yamamoto K, Kanamura N (1987) Comparison of toxicity to plants among $\mathrm{Al}^{3+}$, 
$\mathrm{AlSO}_{4}{ }^{+}$, and $\mathrm{Al}-\mathrm{F}$ complex ions. Soil Sci Plant Nutr 33, 43-55

Tang Van Hai, Truong Thi Nga, Laudelout $H$ (1989) Effect of aluminium on the mineral nutrition of rice. Plant Soil 114, 173-185

Tang Van Hai, Houben V, Mbouti CN, Dufey JE (1993) Diagnostic précoce de la résistance de cultivars de riz (Oryza sativa $\mathrm{L}$ ) à la toxicité aluminique. agronomie 13, 853-860

Taylor GJ (1991) Current views of the aluminium stress response; the physiological basis of tolerance. Curr Top Plant Biochem 10, 57-93

Tran DV (1991) Main issues on rice production on wet acid soils of the Tropics. In : Rice Production on Acid Soils of the Tropics ( $\mathrm{P}$ Deturk, FN Ponnamperuma, eds), Institute of Fundamental Studies, Kandy, Sri Lanka, 87-95

Uwasawa M, Sangtong P, Cholitkul W (1988) Behavior of phosphorus in paddy soils of Thailand. I. Content of inorganic, and available soil $P$ in relation to rice plant P-nutrition. Soil Sci Plant Nutr 34, 41-53 van Breemen N, Moorman FR (1978) Iron-toxic soils. In: Soils and Rice, The International Rice Research Institute IRRI, Los Baños, Philippines, 781-799

Vo Dinh Quang, Dufey JE (1995) Effect of temperature and flooding duration on phosphate sorption in an acid sulphate soil from Vietnam. Eur J Soil Sci 46 , sous presse

Vo Dinh Quang, Tang Van Hai, Dufey JE (1995) Effect of temperature on rice growth in nutrient solution and in acid sulphate soils from Vietnam. Plant Soil (sous presse)

Wagatsuma $\mathrm{Y}$, Ezoe $\mathrm{Y}$ (1985) Effect of $\mathrm{pH}$ on ionic species of aluminium in medium and on aluminium toxicity under solution culture. Soil Sci Plant Nutr 31, 547-561

Watanabe FS, Olsen SR (1965) Test of an ascorbic acid method for determining phosphorus in water and $\mathrm{NaHCO}_{3}$ extracts from soil. Soil Sci Soc Am Proc 29, 677-678

Yoshida S, Forno DA, Cock JH, Gomez AA (1976) Laboratory Manual for Physiological Studies of Rice. The International Rice Research Institute IRRI, Los Baños, Philippines 